\title{
Presencia de Salmonella spp. en un área del Caribe colombiano: un riesgo para la salud pública
}

\author{
Johnny Durango, Germán Arrieta, Salim Mattar \\ Instituto de Investigaciones Biológicas del Trópico, Facultad de Medicina Veterinaria y Zootecnia, \\ Universidad de Córdoba, Montería, Córdoba, Colombia.
}

Salmonella está asociada frecuentemente con la enfermedad diarreica aguda; la bacteria se propaga principalmente por la ingestión de alimentos o de aguas contaminadas o por personas infectadas que manipulan los alimentos. En Colombia no se conoce la distribución de los serotipos de Salmonella en los alimentos. El objetivo de este estudio fue el de establecer la frecuencia de Salmonella spp. en alimentos del Caribe colombiano. Se analizaron 636 muestras de alimentos obtenidas en ventas de comidas rápidas callejeras y en plazas de mercados de Barranquilla $(n=245)$, Montería $(n=222)$, Sincelejo $(n=87)$ y Cartagena $(n=82)$. El aislamiento de la bacteria se realizó por el método convencional de la Food and Drug Administration (FDA) de los Estados Unidos. Se inocularon $25 \mathrm{~g}$ de cada muestra de alimento en $225 \mathrm{ml}$ de caldo de preenriquecimiento; posteriormente, se inoculó $1 \mathrm{ml}$ a los caldos de enriquecimiento y, finalmente, se sembraron en medios de cultivo selectivos para Salmonella. Las colonias sospechosas se identificaron por pruebas bioquímicas y serológicas convencionales para Salmonella. Se aislaron 47 (7,4\%) Salmonella spp. Del total de muestras de carne de res, 9,3\% fueron positivas para Salmonella spp., 12,6\% de chorizo, 7,9\% de queso, $5,2 \%$ de carne de cerdo, $1,6 \%$ de pollo y 10,5\% de arepa de huevo. Los principales serotipos encontrados fueron S. Anatum (26\%), S. Newport (13\%), S. Typhimurium (9\%), $S$. Gaminara (9\%) y $S$. Uganda (9\%). Este estudio permitió establecer la distribución de los serotipos de Salmonella spp. en alimentos de la Costa Atlántica colombiana. No se observaron diferencias estadísticas significativas entre los estratos 1 y 4 , ni entre los estratos 2 y 3 $(p>0,05)$, pero sí entre los estratos 1,2 y $3(p<0,05)$. Se debe continuar con la vigilancia de los alimentos para establecer con mayor precisión la importancia de Salmonella spp. en la salud pública del Caribe colombiano.

Palabras clave: Salmonella, alimentos, serotipos, Colombia, Caribe, epidemiología.

\section{Presence of Salmonella as a risk to public health in the Caribbean zone of Colombia}

Salmonella is frequently involved in diarrhoeal disease throughout the world and is disseminated mainly by food, polluted waters or infected food-handlers. In Colombia, the serotypes of Salmonella and their distribution in food have not been characterized. Therefore, the objective was to establish the epidemiology of Salmonella in the Caribbean zone. Six hundred thirty-six samples were obtained in fast food outlets located in city squares or markets of Barranquilla $(n=245)$, Montería $(n=222)$, Sincelejo $(n=87)$ and Cartagena $(n=82)$. Salmonella was isolated by the conventional methods recommended by the United States Food and Drug Administration. Briefly, $25 \mathrm{~g}$ of each sample was inoculated in $225 \mathrm{ml}$ of broth. Twenty-four hours later, a $1 \mathrm{ml}$ aliquot was inoculated onto selective media for Salmonella. Suspicious colonies were identified by conventional biochemical tests and confirmed by conventional serology for Salmonella detection. Forty-seven Salmonella serotypes were isolated from meat $(40 \%)$, sausage $(25 \%)$, cheese $(13 \%)$, pig (13\%), chicken $(4.2 \%)$ and egg 'arepas' $(4.2 \%)$. The serologic characterization indicated the following serotypes: S. Anatum (26\%), S. Newport (13\%), S. Typhimurium (9\%), S. Gaminara $(9 \%)$ and S. Uganda (9\%). No statistically significant Salmonella isolations among 4 socioeconomic categories were observed $(p=0.05)$. However, 
differences were observed when rates were compared for Salmonella by food type for socioeconomic categories 1,2 and $3(p<0.05)$, categories 2 and 3 did not show differences between them ( $p>0.05)$.

Key words: Salmonella, food poisoning, Caribbean Colombia, serotypes.

Salmonella spp. es una de las bacterias más complejas de la familia Enterobacteriaceae, con más de 2.400 serotipos. El género Salmonella se clasifica actualmente en dos especies: Salmonella enterica y Salmonella bongori. S.enterica está dividida en 6 subespecies: Salmonella enterica subespecie enterica, Salmonella enterica subespecie salamae, Salmonella enterica subespecie arizonae, Salmonella enterica subespecie diarizonae, Salmonella enterica subespecie houtenae y Salmonella enterica subespecie indica (1).

Salmonella, en el ámbito mundial, está asociada con la enfermedad diarreica aguda (EDA), la cual continúa siendo una de las causas más importantes de morbilidad y mortalidad sobre todo en lactantes, niños y ancianos. Se ha estimado que en Asia, África y Latinoamérica, la probabilidad de que un niño muera por enfermedad diarreica antes de los 7 años pueda llegar al 50\%, dependiendo de los factores socioeconómicos y nutricionales (2). Las infecciones agudas del tracto gastrointestinal están consideradas como una de las más frecuentes en Colombia y causa un mayor número de años perdidos de vida saludable (AVISAS), especialmente en los niños menores de 5 años (3).

La infección por Salmonella spp. está asociada con la ingestión de alimentos preparados o manipulados inapropiadamente o previamente contaminados. Las carnes, los productos lácteos y los huevos crudos son las fuentes más probables de infección o de contaminación extraintestinal focal (4).

Se reportan anualmente, aproximadamente, 50.000 casos de infecciones por Salmonella en el mundo, lo cual representa el $10 \%$ de todas las

\section{Correspondencia:}

Salim Mattar, Km. 26 Vía Ciénaga de Oro. Tel. 094-7560710. Fax: 0947560710

Recibido: 25/06/03; aceptado: 05/03/04 infecciones humanas (5). La bacteria se propaga por la ingestión de alimentos o de aguas contaminadas o por personas infectadas que manipulan el alimento. Se requiere un inóculo de $10^{6}$ a $10^{8}$ bacterias de Salmonella spp. para el desarrollo de la enfermedad sintomática. La dosis de infección que se requiere para producir la enfermedad es menor en personas inmunosuprimidas o con una enfermedad de base como leucemia o linfoma y en personas con acidez gástrica reducida (5). El microorganismo se multiplica a una alta densidad cuando encuentra las condiciones apropiadas como alimentos contaminados o refrigerados inadecuadamente.

En Colombia, existe un sistema de vigilancia por el laboratorio de EDA (6), el cual ha permitido conocer los serotipos más importantes implicados en esta enfermedad, pero no se conocen los serotipos de Salmonella ni su distribución en los alimentos; de ahí, la importancia de este estudio cuyo objetivo fue conocer la frecuencia de aislamiento de Salmonella spp. en alimentos del Caribe colombiano.

\section{Materiales y métodos}

\section{Ciudades estudiadas}

Durante el 2002, se analizaron 636 muestras de alimentos de comidas rápidas provenientes de ventas callejeras tipo frituras, restaurantes y plazas de mercados. Las 636 muestras fueron tomadas de sitios diferentes de cuatro ciudades de la costa norte colombiana, distribuidas así: Barranquilla $(n=245)$, Montería $(n=222)$, Sincelejo $(\mathrm{n}=87)$ y Cartagena $(\mathrm{n}=82)$.

\section{Tamaño de la muestra}

Para calcular el tamaño de la muestra, se tuvieron en cuenta los datos suministrados por las Cámaras de Comercio de las ciudades estudiadas que informaron que, hasta el 2001, tenían inscritos 1.300 restaurantes en Barranquilla, 700 en Cartagena, 300 en Montería y 200 en Sincelejo. 
Pero, debido a la alta migración del campo, el desplazamiento de poblaciones que terminan ejerciendo la venta de alimentos incontrolada y el poco control que se ejerce en los puestos de ventas de los mercados, no existen datos recientes por parte del Departamento Nacional de Estadísticas (Dane) que permitan saber con certeza el número de ventas ambulantes que se movilizan en los diferentes puntos de las ciudades, por lo que se cree que existe un subregistro de estas ventas. Debido a esto, fue necesario incrementar en $10 \%$ el número de sitios de expendio en cada ciudad y quedó un universo de 1.460 en Barranquilla, 770 en Cartagena, 330 en Montería y 220 en Sincelejo, para un total de 2.780 establecimientos en las cuatro ciudades.

Dadas las escasas medidas de control para la venta de alimentos en la Costa Atlántica, se esperaba una frecuencia alta de contaminación, pero esto disminuía el número de alimentos por analizar. Por lo tanto, para calcular el tamaño de la muestra se tomaron los siguientes parámetros: frecuencia esperada de contaminación alimentaria del $4 \%$; error máximo aceptable del $1 \%$, y nivel de confianza del 99\% (Epi-Info,versión 6) para un tamaño de 720 muestras; sin embargo, se sólo se obtuvieron 636 muestras.

Tipo de muestreo, condiciones de los sitios de muestreo y ubicación

Se llevó a cabo un muestreo al azar con observación de los siguientes aspectos: composición y estado del alimento, condiciones higiénicas del local de expendio y estrato socioeconómico donde estaba situado.

Por composición, se tuvieron en cuenta los alimentos que consistían en productos de origen animal como carne, huevo, leche y sus derivados. En cuanto al estado, se clasificaron en crudos y cocidos.

Los establecimientos se categorizaron en tres niveles y no se tuvo en cuenta el número de empleados por establecimiento: nivel 1, conformado por establecimientos con buenas características sanitarias, presencia de una fuente de suministro de agua, lavadero, buen manejo de basuras (canecas, bolsas para desechos), uso de guantes y gorros, y aislamiento total del alimento del medio ambiente; nivel 2, establecimientos con regulares características sanitarias, escasa disposición de agua, fuentes limitadas de agua, recipientes para el almacenamiento, mal manejo de basuras (con canecas en mal estado sin bolsas para desechos), algunos con guantes y sin gorros, aislamiento parcial del alimento del medio ambiente; $y$ el nivel 3, establecimientos con malas características sanitarias, carencia de agua en el establecimiento y mal manejo de basuras (sin canecas ni bolsas para desechos), sin uso de guantes ni gorros y sin aislamiento del alimento del medio ambiente. Por otro lado, con el fin de evitar posibles sesgos de información en el estudio, la categorización de los establecimientos se realizó con criterios (instrumentos) rigurosos y previamente estandarizados por dos observadores.

El muestreo se llevó a cabo en diferentes puntos de venta comercial y callejera de distintos barrios de las ciudades en estratos 1, 2, 3 y 4; el estrato 1 fue considerado el más bajo.

\section{Análisis microbiológico de los alimentos}

Una vez tomados los alimentos del sitio de expendio, se trasladaron refrigerados al laboratorio del Instituto de Investigaciones Biológicas del Trópico (IIBT) de la Universidad de Córdoba para ser procesados antes de 24 horas.

El aislamiento de Salmonella se realizó por el método de la FDA (7), modificado al adicionarse caldo infusión cerebro corazón (BHI) como medio de preenriquecimiento; se pesaron $25 \mathrm{~g}$ de cada muestra y se inocularon en $225 \mathrm{ml}$ de los medios de preenriquecimiento, agua peptonada y $\mathrm{BHI}$, los cuales se incubaron a $37^{\circ} \mathrm{C}$ durante 24 horas; a partir de éstos, se inoculó $1 \mathrm{ml}$ de cada muestra en $9 \mathrm{ml}$ de caldo Rappaport y tetrationato; posteriormente, se subcultivaron en agar XLT4 (Difco, Detroit, MI USA), SMID (Biomeriux, Marcy L'Etoile, France), SS, Hekctoen, XLD y bismuto sulfito los cuales se incubaron a $37^{\circ} \mathrm{C}$ por 24 horas. Las colonias sospechosas de tener Salmonella se identificaron con pruebas bioquímicas convencionales. La identificación serológica la realizó el Grupo de Microbiología del Instituto Nacional de Salud, con el esquema de KauffmanWhite (8). 


\section{Análisis de los resultados}

Para el análisis de las significancias de las diferencias encontradas en los establecimientos de ventas de alimentos y la frecuencia de gérmenes encontrados entre las ciudades se utilizó la prueba de ji al cuadrado para proporciones.

\section{Resultados}

Se tomaron 636 muestras de alimentos, de las cuales, $204(32 \%)$ eran de carne de res, 95 (15\%) de chorizo, $76(12 \%)$ de queso, $115(18 \%)$ de carne de cerdo, $127(20 \%)$ de pollo y $19(3 \%)$ de arepa de huevo; 471 (74\%) eran de alimentos crudos y $165(26 \%)$ eran de alimentos cocidos. Al estratificar las muestras por categoría sanitaria del establecimiento, se encontró que, de las 636 muestras, $64(10 \%)$ eran de establecimientos del nivel 1, $190(30 \%)$ del nivel 2 y $382(60 \%)$ del nivel
3. Del total de las muestras, $190(30 \%)$ eran procedentes de establecimientos situados en zonas de estrato $1,160(25 \%)$ de estrato 2,242 (38\%) de estrato 3 y 44 (7\%) de estrato 4.

De las 636 muestras estudiadas, se aisló Salmonella spp. en 47 (7,4\%). Al analizar por tipo de alimento, se encontró que 19/204 (9,3\%) muestras de carne de res fueron positivas para Salmonella spp., 12/95 (12,6\%) de chorizo, 6/76 $(7,9 \%)$ de queso, $6 / 115(5,2 \%)$ de carne de cerdo, $2 / 127(1,6 \%)$ muestras de pollo y $2 / 19(10,5 \%)$ arepas de huevo. En el cuadro 1 se encuentra la distribución de los aislamientos de Salmonella spp. por ciudad y por composición del alimento; $31 / 471(6,6 \%)$ de los alimentos crudos fueron positivos para Salmonella spp., especialmente de carne de res, $17 / 31$ (55\%), y 16/165 (9,7\%) de alimentos cocidos, de los cuales $7 / 16(43,8 \%)$ eran de chorizo (cuadro 2).

Cuadro 1. Distribución geográfica y por tipo de alimento de los aislamientos de Salmonella spp.

\begin{tabular}{lrccccccc}
\hline Ciudad & $\mathbf{n}$ & $\begin{array}{c}\text { Carne de } \\
\text { res }\end{array}$ & Chorizo & Queso & Cerdo & Pollo & $\begin{array}{c}\text { Arepa de } \\
\text { huevo }\end{array}$ & Total \\
& & & & $\mathbf{n}(\%)$ & & & \\
\hline Barranquilla & 245 & $9(3,6)$ & $1(0,4)$ & $1(0,4)$ & $2(1,4)$ & 0 & $1(0,4)$ & $14(5,7)$ \\
Cartagena & 82 & $3(3,6)$ & 0 & $2(2,4)$ & $1(12)$ & $1(12,5)$ & $1(1,2)$ & $8(9,8)$ \\
Montería & 222 & $6(2,7)$ & $10(4,5)$ & $3(1,3)$ & $3(1,3)$ & $1(4)$ & 0 & $23(10,3)$ \\
Sincelejo & 87 & $1(1,1)$ & $1(1,1)$ & 0 & 0 & 0 & 0 & $2(2,3)$ \\
Total & $\mathbf{6 3 6}$ & $\mathbf{1 9 ( 2 , 9 )}$ & $\mathbf{1 2 ( 1 , 8 )}$ & $\mathbf{6 ( 0 , 9 )}$ & $\mathbf{6 ( 0 , 9 )}$ & $\mathbf{2}(\mathbf{0 , 3})$ & $\mathbf{2}(\mathbf{0} \mathbf{( 0 , 3 )}$ & $\mathbf{4 7}(\mathbf{1 0 0})$ \\
\hline
\end{tabular}

Cuadro 2. Distribución de los aislamientos de Salmonella spp, por clase de alimento: cocidos o crudos.

\begin{tabular}{|c|c|c|c|c|}
\hline \multirow[t]{2}{*}{ Ciudad } & \multirow[t]{2}{*}{$\mathrm{n}$} & $\begin{array}{c}\text { Tipo de alimento } \\
\text { cocido }\end{array}$ & $\begin{array}{c}\text { Tipo de alimento } \\
\text { crudo }\end{array}$ & \multirow[t]{2}{*}{$\begin{array}{c}\text { Total } \\
\text { Salmonella n (\%) }\end{array}$} \\
\hline & & & & \\
\hline Barranquilla & 245 & $\begin{array}{rr}\text { Chorizo } & (1) \\
\text { Huevo } & (1) \\
\text { Cerdo } & (2)\end{array}$ & $\begin{array}{r}\text { Carne de res }(9) \\
\text { Cerdo }(\text { ) } \\
\text { Queso }(1)\end{array}$ & $14(5,7 \%)$ \\
\hline Cartagena & 82 & $\begin{aligned} \text { Huevo } & (1) \\
\text { Queso } & (1) \\
\text { Cerdo } & (1) \\
\text { Pollo } & (1)\end{aligned}$ & $\begin{array}{r}\text { Carne de res } \\
\text { Queso }\end{array}$ & $8(9,8 \%)$ \\
\hline Montería & 222 & $\begin{array}{rr}\text { Chorizo } & (6) \\
\text { Queso } & (1) \\
\text { Cerdo } & (1) \\
\text { Carne de res } & (2)\end{array}$ & $\begin{array}{rr}\text { Chorizo } & (4) \\
\text { Queso } & (2) \\
\text { Cerdo } & (2) \\
\text { Pollo } & (1) \\
\text { Carne de res } & (4)\end{array}$ & $23(10,3 \%)$ \\
\hline $\begin{array}{l}\text { Sincelejo } \\
\text { Total }\end{array}$ & $\begin{array}{r}87 \\
636\end{array}$ & $\begin{array}{r}\text { Chorizo } \\
16\end{array}$ & $\begin{array}{r}\text { Carne de res } \\
\mathbf{3 1}\end{array}$ & $\begin{array}{c}2(2,3) \\
47(100 \%)\end{array}$ \\
\hline
\end{tabular}


Se observó que $97 \%$ de los establecimientos en donde se aisló Salmonella spp. tenía malas condiciones sanitarias (nivel 3). No se encontró diferencia estadística significativa en las variables de mal manejo de basuras, escasa disposición de agua y alimentos expuestos a diversas fuentes de contaminación ( $p>0,05)$.

De los 190 alimentos analizados en el estrato 1 , en $33(17 \%)$ se aisló Salmonella spp., en 5/160 (3\%) del estrato 2 , en $4 / 242(1,7 \%)$ del estrato $3 y$ en $5 / 44(11 \%)$ del estrato 4 . No se observaron diferencias estadísticas significativas entre los estratos 1 y 4 , ni entre el 2 y el $3(p>0,05)$, pero sí entre los estratos 1,2 y $3(p<0,05)$.

El análisis de la distribución por ciudad de los alimentos contaminados con Salmonella spp. no mostró diferencias estadísticamente significativas, con la excepción de Sincelejo cuando se comparó con Montería ( $p=0,019)$ (cuadro 3).

La tipificación serológica arrojó diversos serotipos con una distribución porcentual variada; resalta la alta frecuencia de $S$. Anatum (25,5\%), S. Newport $(12,8 \%), S$. Typhimurium $(8,5 \%), S$. Gaminara $(8,5 \%)$ y $S$. Uganda $(8,5 \%)$. El $36,2 \%$ restante correspondió a 12 serotipos diferentes (cuadro3).

Cuadro 3. Distribución por departamento de los serotipos de Salmonella spp aisladas de alimentos en la costa atlántica de Colombia durante el 2002.

\begin{tabular}{lll}
\hline Ciudad & \multicolumn{1}{c}{ Serotipo } & n (\%) \\
\hline Montería & S. Newport & $5(21,7)$ \\
& S. Anatum & $4(17,4)$ \\
& S. Gaminara & $4(17,4)$ \\
& S. Typhimurium & $3(13,1)$ \\
& S. Infantis & $2(8,7)$ \\
Barranquilla & Otros $^{1}$ & $5(21,7)$ \\
& S. Anatum & $7(50,0)$ \\
& S. Derby & $2(14,3)$ \\
Cartagena & S. Uganda & $2(14,3)$ \\
& Otros ${ }^{2}$ & $3(21,4)$ \\
Sincelejo & S. Muenchen & $2(25,0)$ \\
& S. Isangi & $2(25,0)$ \\
& Otros $^{3}$ & $4(50,0)$ \\
& S. Typhimurium & $1(50,0)$ \\
& S. Anatum & $1(50,0)$ \\
\hline
\end{tabular}

\footnotetext{
S. Uganda, S. Agona, S. Senftnberg, S. Saintpaul, S. Branderbug

${ }^{2}$ S. Senftnberg, S. Sandiego y Salmonella spp.

${ }^{3}$ S. Duesseldorf, S. Newport, S. Rubislaw, S. Uganda
}

\section{Discusión}

El estudio mostró por vez primera en el Caribe colombiano los serotipos de Salmonella aislados de los alimentos contaminados. En Colombia existen muy pocas publicaciones sobre los serotipos de Salmonella encontrados en alimentos. El conocimiento de estos serotipos es de gran importancia en salud pública ya que permitirá la intervención para controlar y vigilar las salmonelosis. El país no cuenta con una epidemiología de las toxiinfecciones alimentarias, debido a que los hospitales y los centros de salud regionales no tienen la infraestructura necesaria para determinar la etiología y realizar la vigilancia epidemiológica de los brotes. Además, un sinnúmero de casos permanecen subregistrados ya que muchos pacientes no acuden a los centros de salud y son tratados ambulatoriamente por médicos generales que no reportan los casos. Este problema epidemiológico también se presenta en los Estados Unidos con un subregistro apreciable de casos (5).

Los alimentos analizados fueron principalmente productos a base de carne de res, cerdo, pollo y queso; de éstos, $66 \%$ eran crudos y $34 \%$ tenían algún tipo de cocción. De la carne de res $(9,3 \%)$ que fue positiva para Salmonella, el $10 \%$ era cocida y el $90 \%$ cruda; de los chorizos ( $25 \%$ ), el $66 \%$ estaban cocidos y el $34 \%$ crudos. El chorizo, la arepa de huevo y la carne de res, principalmente molida, fueron los alimentos con mayor contaminación por Salmonella spp., relacionado posiblemente con la excesiva manipulación y la variedad de ingredientes que se usa en su preparación. El queso y la carne de cerdo también presentaron un alto grado de contaminación con Salmonella spp.; la alta frecuencia de contaminación $(43,8 \%)$ de los alimentos cocidos evidencia el riesgo potencial de infección en este tipo de alimentos, debido posiblemente al mismo factor de manipulación. Si se tiene en cuenta al chorizo como un producto cárnico de diversas fuentes, se tendría que el $83 \%$ de los alimentos contaminados con Salmonella spp. fueron alimentos a base de diversos tipos de carne (cuadro 1).

El desplazamiento de personas del campo a las ciudades, el desempleo y las pocas medidas de 
control en las plazas de mercado y sitios de expendio han generado una proliferación de puestos de ventas de alimentos callejeros con pocas medidas de higiene y control sanitario totalmente expuestos a la contaminación, lo cual pone en riesgo la salud de las personas.

Nuestros hallazgos coinciden con los del Laboratorio Nacional de Referencia de Salmonella y Shigella de España (9), donde el mayor número de cepas se aisló de alimentos de productos cárnicos como embutidos, carne molida, pollo y, en general, carnes procesadas y manipuladas en exceso. Con respecto a la distribución de los serotipos, el predominio en España fue de Enteritidis, Typhimurium, Hadar y Virchow (9), mientras que en nuestro estudio predominaron los serotipos Anatum, Newport, Typhimurium y Gaminara. En un estudio preliminar reciente demostramos por la técnica de rep-PCR que estas cepas se parecen entre sí y que, posiblemente, existen un par de conglomerados que circulan en el Caribe colombiano; no obstante, se debe utilizar otra técnica como PFGE o ribotipificación para confirmar este hallazgo (10).

Nuestros resultados coinciden también con los obtenidos por el Laboratorio Nacional de Salud Pública de México, que reportó que un $41 \%$ de las muestras de chorizo resultaron contaminadas por Salmonella (11) y un aislamiento de $4,42 \%$ de Salmonella en chorizos crudos de una fábrica procesadora. Además, se informó un $0,84 \%$ de aislamientos en superficies vivas y de $0,9 \%$ en materias fecales de los operadores de la misma empresa.

Al comparar nuestro porcentaje de aislamiento de Salmonella spp. $(7,4 \%)$ con el encontrado por Ovalle et al. (12) en Bogotá (8\%), se observaron resultados muy similares $(p>0,05)$. A pesar de las diferencias geoclimáticas y de control sanitario que se da en Bogotá y la Costa Caribe, también reportaron aislamientos de cepas de Salmonella spp. a partir del chorizo de cerdo. En la vigilancia que se realiza con los laboratorios de salud pública, se encontraron tres serotipos diferentes a los nuestros (Panamá, Muenster y Enteritidis) $(6,13)$. Llama la atención no encontrar en nuestro trabajo el serotipo Enteritidis que se presume es el más prevalente en la toxiinfección alimentaria. Sin embargo, en los resultados presentados por el INS, no hay aislamientos de la Costa Caribe colombiana lo que explicaría su distribución variable y contradictoria en los trabajos.

En otro estudio preliminar nuestro (14), de 144 muestras de alimentos analizados, se obtuvieron tres aislados de Salmonella spp. (2\%) de muestras de carne de cerdo crudo, arepa de huevo y chorizo.

Los alimentos a base de pollo, $2(1,6 \%)$, resultaron positivos para Salmonella spp.; estos hallazgos coinciden con el trabajo de Ovalle et al. (12), quien reportó aislamientos de Salmonella spp. en alimentos a base de pollo en Bogotá. En nuestro estudio, también se aisló Salmonella spp. de alimentos donde parte de su contenido era a base de huevo y productos lácteos; sin embargo, fue imposible determinar bajo las condiciones del estudio, si la contaminación observada provenía de los productos o del manipulador. Además de la inapropiada manipulación de los alimentos, una de las características generales encontradas en los puestos callejeros de venta de alimentos durante este estudio fue la mala disposición de agua que presentaron; en estos sitios no existían fuentes constantes de agua como lavabos, lavamanos, etc..

Los establecimientos también presentaron una deficiente o mala disposición de basuras que puede ser una posible fuente de contaminación, ya que la acumulación de los residuos líquidos y sólidos es un ambiente propicio para la llegada de vectores como moscas y roedores.

Al comparar la frecuencia de contaminación por Salmonella entre las ciudades no existen diferencias entre ellas $(p>0,05)$, con la excepción de Sincelejo, probablemente debido a la baja casuística $(n=87)$. No obstante, Cartagena $(n=82)$ mostró una alta frecuencia de contaminación $(9,8 \%)$, lo cual puede deberse al tipo de establecimiento que se tomó en cada ciudad.

Con respecto a la distribución de Salmonella en los estratos estudiados, se observó una diferencia estadística significativa entre los estratos 1,2 y $3(p<0,05)$. A pesar de que se encuentran en una región con diferencias sociales acentuadas, curiosamente no se observaron diferencias estadísticas entre los estratos 1 y $4(p>0,05)$. Sin 
embargo, este hallazgo se debe posiblemente a la baja casuística del estrato 4 que fue de sólo 44 muestras. Tampoco deberían hallarse diferencias entre los estratos 3 y 4 , ya que tienen condiciones socioeconómicas similares, pero el análisis estadístico demostró que sí existieron diferencias $(p<0,05)$, probablemente por el bajo muestreo en el estrato 4.

La tasa de contaminación por coliformes observada en este estudio fue muy alta (98\%) (datos no mostrados) y está de acuerdo con lo observado con las condiciones sanitarias de los establecimientos analizados. Estos resultados coinciden con los reportados por Ovalle et al. (12), quien determinó una contaminación por coliformes del $72 \%$ en los alimentos de Bogotá.

En conclusión, los alimentos manipulados inadecuadamente son uno de los principales medios de contaminación por Salmonella, de ahí, la importancia de seguir la evolución de los serotipos en brotes que afectan algunas regiones y el país. Este trabajo estableció la epidemiología preliminar de Salmonella en los alimentos de la Costa Atlántica colombiana; se requiere continuar y aumentar el análisis de los alimentos para establecer con mayor precisión su magnitud en la salud pública de los habitantes del Caribe colombiano; el fenómeno de ventas callejeras de alimentos manipulados inadecuadamente es un riesgo para la salud de la población que los consume, por lo que se aconseja a las autoridades competentes tener un mayor control sanitario sobre los mismos.

\section{Agradecimientos}

A Colciencias, proyecto 1112-04-10182, Ct. 2492000 y al Comité Central de Investigaciones de la Universidad de Córdoba por su apoyo financiero, dentro del marco del proyecto río Sinú, a este importante proyecto regional. Al Grupo de Microbiología del INS por la serotipificación de los aislamientos de salmonela.

\section{Referencias}

1. Popoff $\mathbf{M}$, Le Minor $\mathbf{L}$. Formules antigéniques de sérovars de Salmonella. Paris: Centre Colalorateur OMS de Réference et de Recherches pour les Salmonella; 1997. p.151.
2. Bern C, Martines J, De Zoysa I, Glass RI. The magnitude of the global problem of diarrhoeal disease: a ten year update. Bull World Health Org 1992;70:705-14.

3. Rodríguez J, Gallardo HM. Mortalidad y años de vida ajustados por discapacidad como medidas de la carga de enfermedad. Colombia 1985-1995. Bogotá: Ministerio de Salud; 1999.

4. Gast R, Porter R. Applying test for specific yolk antibodies to predict contamination by Salmonella enteritidis in eggs from experimentally infected laying hens. Avian Dis 1997;30:195-202.

5. Mead PS, Slutsker L, Dietz V, McCaig L, Bresee J, Shapiro C, Griffin P, Tauxe R. Food-related illness and death in the United States. Emerg Infect Dis 1999;5: 607-25.

6. Muñoz N, Agudelo Cl, Ovalle MV, Realpe MH y coordinadoras del programa en los Laboratorios de Salud Pública. Vigilancia en red de la susceptibilidad antimicrobiana y de los serotipos de Salmonella spp., Shigella spp. y Vibrio cholerae O1, 2000-2001. Inf Quinc Epidemiol Nac 2002;7:184-8.

7. Food and Drug Administration. Food and Drug Administration/AOAC BAM 2003; http://www.cfsan. fda.gov/ ebam/bam-toc.htm

8. Popoff MY, Le Minor L. Formules antigeniques des serovars des Salmonella. WHO collaborating Centre for Reference and Research on Salmonella. Paris: Institut Pasteur; 2001.

9. Ministerio de Sanidad y Consumo de España. Infecciones por Salmonella notificadas al sistema de información microbiológica. Boletín epidemiológico de España 2001;9.

10. Máttar S, Durango J, Moreno C, Arrieta G. Epidemiology of Salmonella strains food-related in the Caribbean area of Colombia. 103 ASM $^{\text {th }}$ Meeting, American Society for Microbiology, May 18-22, 2003. Washington, D.C.: ASM; 2003. p.142.

11. Salgado J, Jaramillo C, Nunes F. Salmonella spp. en tres tipos de chorizos, como peligro dentro de un sistema de análisis de riesgo e identificación de puntos críticos de control (HACCP), en una empacadora de la ciudad de México. Vet Mex 1999;30:157-65.

12. Ovalle $\mathbf{Y}$, Reyes $\mathbf{Y}$, Máttar S. Aislamiento y serotipificación de Salmonella spp. a partir de alimentos obtenidos en diferentes zonas de Santa Fé de Bogotá (tesis). Bogotá: Pontificia Universidad Javeriana; 1999.

13. Muñoz N, Agudelo Cl, Ovalle MV, Realpe MH y coordinadoras del programa en los Laboratorios de Salud Pública. Vigilancia en red de la susceptibilidad antimicrobiana y de los serotipos de Salmonella spp., Shigella spp. y Vibrio cholerae 01, 1997-1999. Biomédica 2000;20:210. 
14. Carrascal A, Arrieta G, Máttar S. Estudio preliminar de la calidad microbiológica de los alimentos en la Costa Atlántica colombiana. Inf Quinc Epidemiol Nac 2002;7: 163-9.
15. Craven S, Williams D. Inhibition of Salmonella attachment to chicken cecal mucus by intestinal isolates of Enterobacteriaceae and Lactobacilli. Avian Dis 1997;30: 548-57. 